\title{
Dietary patterns and suicide in Japanese adults: the Japan Public Health Center-based Prospective Study
}

Akiko Nanri, Tetsuya Mizoue, Kalpana Poudel-Tandukar, Mitsuhiko Noda, Masayuki Kato, Kayo Kurotani, Atsushi Goto, Shino Oba, Manami Inoue, Shoichiro Tsugane, for the Japan Public Health Center-based Prospective Study Group*

\section{Background}

Although dietary patterns have been linked to depression, a frequently observed precondition for suicide, no study has yet examined the association between dietary patterns and suicide risk.

\section{Aims \\ To prospectively investigate the association between dietary patterns and death from suicide.}

\section{Method}

Participants were 40752 men and 48285 women who took part in the second survey of the Japan Public Health Centerbased Prospective Study (1995-1998). Dietary patterns were derived from principal component analysis of the consumption of 134 food and beverage items ascertained by a food frequency questionnaire. Hazard ratios of suicide from the fourth year of follow-up to December 2005 were calculated.

\section{Results}

Among both men and women, a 'prudent' dietary pattern characterised by a high intake of vegetables, fruits, potatoes, soy products, mushrooms, seaweed and fish was associated with a decreased risk of suicide. The multivariable-adjusted hazard ratio of suicide for the highest $v$. lowest quartiles of the dietary pattern score was $0.46(95 \% \mathrm{Cl}$ 0.28-0.75) $(P$ for trend, 0.005). Other dietary patterns (Westernised and traditional Japanese) were not associated with suicide risk.

\section{Conclusions}

Our findings suggest that a prudent dietary pattern may be associated with a decreased risk of death from suicide.

\section{Declaration of interest}

None.
The worldwide suicide rate has increased by $60 \%$ over the past 45 years, ${ }^{1}$ making it the tenth leading cause of death, ${ }^{2}$ and therefore a globally important public health issue. In Japan, suicide rates (per 100000) were 36.2 in men and 13.2 in women in 2009, ${ }^{3}$ and more than 30000 suicidal deaths have been recorded each year since 1998, ${ }^{4}$ with those aged 40-69 accounting for over half of the total. ${ }^{4}$ Suicide is known to be associated with psychosocial factors, ${ }^{1}$ but epidemiological evidence linking suicide to other environmental factors is limited. As regards diet, a modifiable factor that could influence mental health, a few studies to date have examined specific nutrients and foods including fish and omega-3 fatty acids, ${ }^{5-7}$ coffee, $^{8-10}$ alcohol $^{11-18}$ and tryptophan ${ }^{19}$ in relation to suicide risk.

A great deal of interest has been directed in recent years towards the analysis of dietary patterns in the field of nutritional epidemiology. A dietary pattern is a comprehensive variable that integrates the consumption of several foods or food groups. Given that individuals consume food as part of a diet and not as single items or nutrients, these patterns are expected to have a greater impact on disease risk than any individual item; ${ }^{20}$ further, dietary pattern analysis can clarify the actual effect of the overall consumed diet. Additionally, even in cases where few dietary factors associated with a disease can be identified, such dietary pattern analysis may still prove useful. ${ }^{20}$ Previous studies have reported an inverse association between depression, which is an important factor as a cause of suicide, and dietary patterns characterised by high intake of vegetables, fruits and fish. $^{21-25}$ However, to the best of our knowledge, no study has yet examined the association between overall dietary patterns and suicide risk. We therefore prospectively investigated the association between major dietary patterns and suicide risk, using data from a large-scale, population-based cohort study in Japan.

*See online supplement for details of the study group members

\section{Method}

\section{Study population}

The Japan Public Health Center-based (JPHC) Prospective Study was launched in 1990 and 1993 for cohorts I and II respectively. ${ }^{26}$ Participants in cohort I were residents of five Japanese Public Health Center areas aged 40-59 years and those in cohort II were residents of six other Japanese Public Health Center areas aged 40-69 years. The study sites are scattered across Japan but are mainly in rural areas. The participants were informed of the objectives of the study and those who completed the survey questionnaire were regarded as consenting to participation. This survey was conducted at baseline as well as at 5- (second) and 10-year (third) follow-up sessions. Each survey gathered information on medical history and health-related behaviours, including smoking, drinking and dietary habits. This study was approved by the Institutional Review Board of the National Cancer Center of Japan.

Of the study population at baseline $(n=140420), 103782$ participants responded to the second survey, and of these, 102695 completed the food frequency questionnaire (FFQ) at the second survey. After exclusion of 1065 participants who reported extreme total energy intake (outside of mean plus or minus 3 standard deviations (s.d.), according to gender), 101630 participants (47408 men and 54222 women) were ultimately included in analysis of dietary patterns. We further excluded 12593 participants who did not respond to the baseline survey, leaving a total of 89037 participants (40752 men and 48285 women) ultimately enrolled to assess the association between dietary patterns and suicide risk. Participants who completed the questionnaire at the second survey, compared with those who did not, were less likely, at baseline, to be a smoker, a drinker of alcohol (women only) and physically inactive at leisure (men only). 


\section{Food frequency questionnaire}

Although participants completed self-administered questionnaires at the first, second and third surveys, we used data from the second survey as our baseline, because the questionnaire used for that particular survey contained more comprehensive information on food intake than did the first. At the second survey, an FFQ was used to assess the average intake of 147 food and beverage items over the previous year. ${ }^{27}$ For most food items, nine options were available for describing consumption frequency: rarely (<once/month), 1-3 times/month, 1-2 times/week, 3-4 times/week, 5-6 times/week, once a day, 2-3 times/day, 4-6 times/day, and $\geqslant 7$ times/day. Slightly different options were used for beverage intake. A standard portion size was specified for each food item and the respondents were asked to assess their usual portion size relative to a standard specified for each food item using three options (less than half, standard, more than 1.5 times). Daily intake of most foods was calculated by multiplying the daily consumption frequency and the standard portion size by the individual's usual portion size. The validity and reproducibility of the FFQ had already been established as reasonable. ${ }^{28-30}$

\section{Dietary patterns}

Details regarding the identification of dietary patterns have been described elsewhere. ${ }^{31}$ Briefly, we used 134 food and beverage items of the FFQ to derive dietary patterns. Some foods or food groups that were similar in nutritional content or culinary use were combined, leaving a total of 48 food groups. We performed principal component analysis based on log-transformed intakes of these 48 food groups. The factors were then rotated by orthogonal transformation (varimax rotation) to maintain uncorrelated factors and increased interpretability. We determined three factors with eigenvalues, the scree test and the interpretability of the factors. Dietary patterns were named according to food items that had the highest weight on each of the three factors. The factor scores for each dietary pattern were calculated for each participant by summing the intakes of food items weighted by their factor loadings. The scores were energy-adjusted using the residual method. The validity and reproducibility of the identified dietary patterns have been determined to be acceptable. ${ }^{31}$

\section{Follow-up and outcome}

The participants' residency and vital status were followed up using the residential registry. Causes of death were confirmed via death certificates (with permission) and were defined according to the ICD- $10,{ }^{32}$ the focus of the present study being death from suicide (ICD-10: X60 to X84).

\section{Statistical analysis}

Person-years of follow-up were calculated for each person starting from the date of response to the second survey questionnaire until either the date of death or 31 December 2005, whichever came first. Participants were divided into quartiles of factor scores for each dietary pattern based on the separate distribution for men and women. Confounding variables considered were as follows: age (years, continuous), study area (11 areas), body mass index $\left(<21,21.0-22.9,23.0-24.9,25.0-26.9\right.$ or $\left.\geqslant 27.0 \mathrm{~kg} / \mathrm{m}^{2}\right)$, smoking status (lifetime non-smoker, former smoker or current smoker with a consumption of either $<20$ or $\geqslant 20$ cigarettes/day), total physical activity (metabolic equivalent task h/day, quartiles), history of diabetes mellitus (yes or no), history of hypertension (yes or no), total energy intake (kcal/day), mental stress in daily life (low, medium and high), living status (alone, with spouse only, child(ren)/parent(s) with spouse, child(ren)/ parent(s) without spouse, or other), history of serious disease including cancer, cerebrovascular disease, myocardial infarction, chronic liver disease and renal disease before follow-up (yes or no), and occupation (full-time agriculture/forestry/fishery, fulltime salaried/self-employed/professional, multiple occupations, full-time housework/unemployed or other). Mental stress was assessed by using a single question ('How much stress do you feel in your daily life?') with three options (low, medium and high). An indicator variable for missing data was created for each covariate.

We analysed data from cohort I and cohort II combined because the interaction by cohort type on the association between dietary patterns and suicide risk was not statistically significant. Cox proportional hazard regression analysis was used to estimate hazard ratios and 95\% confidence intervals of mortality from suicide for quartiles of scores for each dietary pattern, taking the lowest quartile category as reference. To minimise a potential influence of mental illness at baseline, we excluded deaths from suicide during the first 3 years of follow-up. The first model was adjusted for age, and the second model was further adjusted for smoking status, total physical activity, history of diabetes mellitus, history of hypertension, total energy intake, mental stress, living status and history of serious disease. Study areas were included as strata in the model. Trend association was assessed by assigning ordinal numbers $(0-3)$ to quartile categories of each dietary pattern. Further, because of the relatively low number of suicides, we also analysed data for men and women combined. Additionally, we analysed the association between dietary patterns and suicide by self-reported mental stress (low, medium or high) at baseline.

We also conducted a sensitivity analysis by further adding educational-level data (junior high school education, high school education or any college or higher education) using the cohort I data, which included this information. We tested the proportional hazard assumption by using the time-dependent covariate method and found no violation of proportionality. All analyses were performed using SAS version 9.1 on Windows.

\section{Results}

\section{Dietary patterns}

We identified three dietary patterns by principal component analysis (online Table DS1). The first factor was a prudent dietary pattern characterised by high intake of vegetables, fruits, soy products, potatoes, seaweed, mushrooms and fish. The second factor was a Westernised dietary pattern characterised by high intake of meat, processed meat, bread, dairy products, coffee, black tea, soft drink, dressing, sauce and mayonnaise. The third factor was a traditional Japanese dietary pattern characterised by high intake of salmon, salty fish, oily fish, seafoods other than fish and pickles. The prudent, Westernised and traditional Japanese patterns respectively accounted for $19.7 \%, 5.4 \%$ and $4.1 \%$ of the variance in food intake in men, and $19.6 \%, 5.5 \%$ and $3.8 \%$ in women. In total, these patterns explained $29.2 \%$ and $28.9 \%$ of the variability in intake in men and women respectively.

\section{Characteristics of study participants}

Characteristics according to the quartile categories of dietary pattern scores are shown in Table 1 . Men and women with a higher score for the prudent dietary pattern were more likely to be older but were less likely than those with lower scores to be smokers or to report high mental stress. Those with a higher score for the prudent dietary pattern were also more likely to have 
Table 1 Characteristics according to quartiles (Q) of dietary pattern scores

\begin{tabular}{|c|c|c|c|c|c|c|}
\hline & \multicolumn{2}{|c|}{ Prudent dietary pattern } & \multicolumn{2}{|c|}{ Westernised dietary pattern } & \multicolumn{2}{|c|}{ Traditional Japanese dietary pattern } \\
\hline & Q1 (low) & Q4 (high) & Q1 (low) & Q4 (high) & Q1 (low) & Q4 (high) \\
\hline \multicolumn{7}{|l|}{ Men } \\
\hline Participants, $n$ & 10188 & 10188 & 10188 & 10188 & 10188 & 10188 \\
\hline Age, years: mean (s.d.) & $55.4(7.8)^{a}$ & $59.9(7.9)$ & $60.1(7.6)$ & $54.3(7.5)$ & $57.8(8.4)$ & $57.6(7.7)$ \\
\hline Body mass index, $\mathrm{kg} / \mathrm{m}^{2}$ : mean (s.d.) & $23.6(3.0)$ & $23.5(2.9)$ & $23.2(2.9)$ & $23.9(2.9)$ & $24.1(3.0)$ & $23.2(2.8)$ \\
\hline Total physical activity, MET h/day: mean (s.d.) & $33.5(6.7)$ & $33.3(6.7)$ & $34.4(6.8)$ & $32.5(6.5)$ & $33.5(6.6)$ & $33.9(6.8)$ \\
\hline Total energy intake, kcal/day: mean (s.d.) & $2306(908)$ & $1950(589)$ & $2174(782)$ & 2187 (729) & 2179 (847) & $2124(658)$ \\
\hline Current smoker, ${ }^{a} \%$ & 56.5 & 33.5 & 45.0 & 45.9 & 38.0 & 51.4 \\
\hline History of hypertension, \% & 17.0 & 24.1 & 24.1 & 16.1 & 21.9 & 21.1 \\
\hline History of diabetes mellitus, \% & 5.7 & 10.2 & 7.9 & 6.7 & 7.7 & 5.7 \\
\hline History of serious disease, ${ }^{b} \%$ & 8.2 & 12.4 & 11.0 & 8.1 & 9.2 & 9.2 \\
\hline Mental stress, ${ }^{a}$ high: \% & 21.0 & 15.9 & 14.2 & 22.5 & 16.4 & 18.0 \\
\hline Living status, ${ }^{a}$ alone: \% & 6.1 & 1.8 & 3.6 & 3.5 & 4.7 & 2.4 \\
\hline Occupation, ${ }^{a}$ housework/unemployed: \% & 6.6 & 16.1 & 12.2 & 7.5 & 11.5 & 9.4 \\
\hline \multicolumn{7}{|l|}{ Women } \\
\hline Participants, $n$ & 12071 & 12072 & 12072 & 12072 & 12071 & 12071 \\
\hline Age, years: mean (s.d.) & $56.7(8.2)$ & $58.6(7.8)$ & $60.6(7.4)$ & $54.2(7.5)$ & $58.5(8.4)$ & $57.5(7.7)$ \\
\hline Body mass index, $\mathrm{kg} / \mathrm{m}^{2}$ : mean (s.d.) & $23.5(3.2)$ & $23.5(3.2)$ & $23.5(3.2)$ & $23.4(3.1)$ & $23.7(3.3)$ & $23.4(3.1)$ \\
\hline Total physical activity, MET h/day: mean (s.d.) & $32.5(5.8)$ & $32.8(5.6)$ & $32.7(5.8)$ & $32.5(5.6)$ & $32.6(5.7)$ & $32.6(5.7)$ \\
\hline Total energy intake, kcal/day: mean (s.d.) & $2025(842)$ & $1681(506)$ & $1875(698)$ & $1886(624)$ & $1866(769)$ & $1767(550)$ \\
\hline Current smoker, ${ }^{a} \%$ & 9.4 & 3.1 & 3.1 & 7.8 & 5.5 & 5.2 \\
\hline History of hypertension, \% & 18.4 & 23.2 & 25.7 & 14.9 & 22.5 & 21.3 \\
\hline History of diabetes mellitus, \% & 3.3 & 4.9 & 5.0 & 2.4 & 3.9 & 4.0 \\
\hline History of serious disease, ${ }^{\mathrm{b}} \%$ & 6.1 & 7.5 & 7.6 & 5.7 & 6.9 & 6.6 \\
\hline Mental stress, ${ }^{a}$ high: \% & 19.4 & 15.7 & 14.2 & 19.8 & 16.9 & 16.7 \\
\hline Living status, ${ }^{a}$ alone: $\%$ & 7.0 & 7.0 & 7.3 & 5.6 & 10.1 & 4.1 \\
\hline Occupation, ${ }^{a}$ housework/unemployed: \% & 28.2 & 41.1 & 35.8 & 31.9 & 35.9 & 31.6 \\
\hline \multicolumn{7}{|c|}{$\begin{array}{l}\text { MET, metabolic equivalent task. } \\
\text { a. Categorical variables that included two or more categories were categorised into two categories for each variable (smoking status: current smoker or others; mental stress: } \\
\text { high and others; living status: alone and others; occupation: full-time housework/unemployed and others). } \\
\text { b. Serious disease including cancer, cerebrovascular disease, myocardial infarction, chronic liver disease and renal disease. }\end{array}$} \\
\hline
\end{tabular}

hypertension and diabetes. However, after adjustment for age, the prevalence of hypertension in the lowest and the highest category of the prudent dietary pattern was $17.2 \%$ and $19.0 \%$ in men and $16.7 \%$ and $19.1 \%$ in women. The corresponding values for diabetes were $5.9 \%$ and $9.4 \%$ in men and $3.1 \%$ and $4.1 \%$ in women. The Westernised dietary pattern among men and women was inversely associated with age, total physical activity and a history of hypertension, diabetes mellitus or serious disease but was positively associated with high mental stress. Further, body mass index tended to be higher in men with an elevated score for the Westernised dietary pattern and lower in men and women with an elevated score for the traditional Japanese dietary pattern.

\section{Dietary patterns and suicide}

Of 249 cases of death by suicide (171 men and 78 women) identified during the 767866 person-years of follow-up (mean 8.6 years), 163 cases (107 men and 56 women) identified after the fourth year of follow-up were used in the present analysis. Hazard ratios of suicide according to quartile categories of each dietary pattern score are shown in Table 2 (see online Table DS2 for a more detailed version of the table which also includes data for men and women combined). Men and women in the highest category of the prudent dietary pattern had a decreased risk of suicide compared with those in the lowest. Among men, the multivariate-adjusted hazard ratio of suicide for the highest $v$. lowest quartile category of the dietary pattern was 0.47 (95\% CI 0.25-0.89, $P$ for trend 0.04). Among women, although an inverse association between the prudent dietary pattern and suicide risk was not significant in the model adjusted for covariate, the hazard ratio of suicide for the highest quartile category was 54\% lower than that for the lowest category.
When data for men and women were combined (Table DS2), the multivariate-adjusted hazard ratios (95\% CI) of suicide for the lowest through the highest quartile category of the prudent dietary pattern score were 1.00 (reference), 0.71 (0.47-1.07), 0.79 (0.531.19 ) and $0.46(0.28-0.75)$ ( $P$ for trend 0.005$)$. In analyses stratified by mental stress, a decreased risk of suicide associated with the highest category of the prudent dietary pattern was observed among participants with low and medium mental stress, but not among those with high mental stress (Table DS2). Further, when we repeated the analysis using cohort I data after adding educational level as covariate, the same inverse association between the prudent dietary pattern and suicide risk was observed (data not shown). Neither the Westernised nor the traditional Japanese dietary patterns were appreciably associated with suicide risk.

\section{Discussion}

\section{Main findings}

In a large-scale, population-based, prospective study among Japanese adults, a prudent dietary pattern characterised by a high intake of vegetables, fruits, soy products, potatoes, seaweed, mushrooms and fish was significantly associated with a decreased risk of suicide in all participants. To our knowledge, this is the first study to examine the association between dietary patterns and suicide risk.

\section{Findings from other studies}

The inverse association reported in the present study between a prudent dietary pattern and suicide risk is in line with previous findings of an inverse association between similar dietary patterns 


\begin{tabular}{|c|c|c|c|c|c|}
\hline & \multicolumn{4}{|c|}{ Quartiles of dietary pattern scores } & \multirow[b]{2}{*}{ Trend $P^{\mathrm{a}}$} \\
\hline & Q1 (low) & Q2 & Q3 & Q4 (high) & \\
\hline \multicolumn{6}{|l|}{ Men } \\
\hline \multicolumn{6}{|l|}{ Prudent dietary pattern } \\
\hline Deaths, $n$ & 35 & 28 & 29 & 15 & \\
\hline Age-adjusted HR (95\% Cl) & 1.00 (ref) & $0.80(0.48-1.31)$ & $0.84(0.51-1.38)$ & $0.47(0.26-0.87)$ & 0.03 \\
\hline Multivariate-adjusted $^{\mathrm{b}} \mathrm{HR}(95 \% \mathrm{Cl})$ & 1.00 (ref) & $0.82(0.49-1.35)$ & $0.86(0.52-1.43)$ & $0.47(0.25-0.89)$ & 0.04 \\
\hline \multicolumn{6}{|l|}{ Westernised dietary pattern } \\
\hline Deaths, $n$ & 27 & 28 & 31 & 21 & \\
\hline Age-adjusted HR (95\% Cl) & 1.00 (ref) & $1.03(0.61-1.76)$ & $1.18(0.69-2.02)$ & $0.86(0.47-1.59)$ & 0.83 \\
\hline Multivariate-adjusted ${ }^{\mathrm{b}} \mathrm{HR}(95 \% \mathrm{Cl})$ & 1.00 (ref) & $1.04(0.61-1.78)$ & $1.22(0.71-2.08)$ & $0.92(0.49-1.70)$ & 0.98 \\
\hline \multicolumn{6}{|l|}{ Traditional Japanese dietary pattern } \\
\hline Deaths, $n$ & 24 & 20 & 27 & 36 & \\
\hline Age-adjusted HR (95\% Cl) & 1.00 (ref) & $0.73(0.37-1.46)$ & $0.88(0.44-1.75)$ & $1.07(0.54-2.12)$ & 0.45 \\
\hline Multivariate-adjusted ${ }^{\mathrm{b}} \mathrm{HR}(95 \% \mathrm{Cl})$ & 1.00 (ref) & $0.74(0.37-1.47)$ & $0.89(0.45-1.77)$ & $1.09(0.55-2.15)$ & 0.44 \\
\hline \multicolumn{6}{|l|}{ Women } \\
\hline \multicolumn{6}{|l|}{ Prudent dietary pattern } \\
\hline Deaths, $n$ & 22 & 11 & 14 & 9 & \\
\hline Age-adjusted HR (95\% Cl) & 1.00 (ref) & $0.51(0.25-1.05)$ & $0.65(0.33-1.27)$ & $0.42(0.19-0.93)$ & 0.04 \\
\hline Multivariate-adjusted ${ }^{\mathrm{b}} \mathrm{HR}(95 \% \mathrm{Cl})$ & 1.00 (ref) & $0.54(0.26-1.13)$ & $0.70(0.35-1.41)$ & $0.46(0.20-1.05)$ & 0.09 \\
\hline \multicolumn{6}{|l|}{ Westernised dietary pattern } \\
\hline Deaths, $n$ & 19 & 17 & 13 & 7 & \\
\hline Age-adjusted HR (95\% Cl) & 1.00 (ref) & $1.07(0.55-2.08)$ & $1.02(0.49-2.14)$ & $0.71(0.28-1.84)$ & 0.59 \\
\hline Multivariate-adjusted ${ }^{\mathrm{b}} \mathrm{HR}(95 \% \mathrm{Cl})$ & 1.00 (ref) & $1.10(0.57-2.15)$ & $1.01(0.48-2.15)$ & $0.71(0.27-1.84)$ & 0.57 \\
\hline \multicolumn{6}{|l|}{ Traditional Japanese dietary pattern } \\
\hline Deaths, $n$ & 11 & 11 & 18 & 16 & \\
\hline Age-adjusted HR (95\% Cl) & 1.00 (ref) & $0.73(0.31-1.71)$ & $1.00(0.46-2.17)$ & $0.74(0.33-1.65)$ & 0.65 \\
\hline Multivariate-adjusted ${ }^{\mathrm{b}} \mathrm{HR}(95 \% \mathrm{Cl})$ & 1.00 (ref) & $0.76(0.33-1.80)$ & $1.03(0.47-2.26)$ & $0.76(0.34-1.72)$ & 0.69 \\
\hline $\begin{array}{l}\text { a. Based on Cox proportional hazards model } \\
\text { b. Adjusted for age (year), body mass index } \\
\text { total physical activity (quartile of metabolic e } \\
\text { stress (low, medium and high), living status ( } \\
\text { occupation (full-time agriculture/forestry/fish } \\
\text { included as strata in the model. }\end{array}$ & $\begin{array}{l}\text { numbers } 0-3 \text { to the } \\
-24.9,25-26.9 \text { or } \geqslant 2 \\
\text { ay), history of diabet } \\
\text { e, child(ren)/parent(s) } \\
\text { ed/self-employed/pr }\end{array}$ & $\begin{array}{l}\text { le categories of each } \\
\mathrm{n}^{2} \text { ), smoking status ( } \mathrm{n} \\
\text { litus (yes or no), histo } \\
\text { spouse, or child(ren)/ } \\
\text { nal, multiple occupat }\end{array}$ & $\begin{array}{l}\text { ary pattern. } \\
\text {, past, current with a } \\
\text { f hypertension (yes or } \\
\text { nt(s) without spouse), } \\
\text { full-time housework/ }\end{array}$ & $\begin{array}{l}\text { mption of }<20 \text { or } \geqslant \\
\text { otal energy intake ( } k c \\
\text { of serious disease } \\
\text { bloyed, or other). Stuc }\end{array}$ & $\begin{array}{l}\text { arettes/day), } \\
\text { ), mental } \\
\text { no), } \\
\text { as were }\end{array}$ \\
\hline
\end{tabular}

and depression, ${ }^{21-25}$ which is a major precondition of suicide. ${ }^{2}$ In a cross-sectional study in elderly people in France, a healthy cluster characterised by high intakes of fruits and vegetables was associated with a lower score on the Center for Epidemiologic Studies Depression Scale than that of participants in other clusters (small eaters, biscuits and snacking, charcuterie and starchy foods, and pizza and sandwiches) among elderly women. ${ }^{25}$ Two studies among workers have respectively reported an inverse association between a whole-food pattern (high intake of vegetables, fruits and fish) and subsequent depression in the $\mathrm{UK}^{21}$ and an inverse association between a healthy Japanese dietary pattern (high intake of vegetables, fruits, soy products and mushrooms) and depression in Japan. ${ }^{24}$ In an Australian study conducted among women aged 20-93 years, a traditional dietary pattern characterised by vegetables, fruits, meat, fish and whole grains was associated with a lower prevalence of depression. ${ }^{23}$ Another study among Norwegian adults also observed an inverse association between a healthy dietary pattern (high intake of vegetables, salads, fruits, rice, pasta, cereals and fish) and depression. ${ }^{22}$ The results reported in these depression studies support our present findings.

\section{Prudent dietary pattern and suicide}

Although the mechanisms linking the prudent dietary pattern to suicide risk are unclear, several explanations have been posited. Fruits and vegetables were major and common contributors to dietary patterns associated with a decreased risk of suicide in the present study, and with a reduced prevalence or risk of depression in previous studies. ${ }^{21-25}$ These foods are rich in nutrients such as folate and antioxidant vitamins (vitamin C and carotene), which have been shown to be associated with decreased prevalence of depressive symptoms. ${ }^{33,34}$ In the present study, both men and women with higher scores for a prudent dietary pattern consumed greater amounts of these nutrients than those with lower scores; the respective mean intakes for the lowest and highest quartile categories of the dietary pattern were 315 and $447 \mu \mathrm{g}$ for folate, 80 and $162 \mathrm{mg}$ for vitamin $\mathrm{C}$ and 1813 and $4646 \mu \mathrm{g}$ for $\beta$-carotene in men and 361 and $467 \mu \mathrm{g}$ for folate, 117 and $189 \mathrm{mg}$ for vitamin $\mathrm{C}$, and 2914 and $5178 \mu \mathrm{g}$ for $\beta$-carotene in women (all $P$ for trend $<0.001$ ). Folate is involved in the metabolism of monoamines such as serotonin in the brain and is hypothesised to protect brain functions by reducing levels of homocysteine, which exerts neurotoxic effects through several mechanisms. ${ }^{35}$ Further, oxidative stress is hypothesised to be caused by reactive oxygen species, and defective antioxidant defences may therefore be involved in the pathophysiology of neuropsychiatric disorders. ${ }^{36}$ In this context, antioxidant vitamins such as vitamin C may help reduce risk of mood disorders. These nutrients or some combination of them may therefore have a beneficial effect on suicide risk.

\section{Other dietary patterns}

Previous depression studies have observed a positive association between depressive symptoms and both processed food (high intake of sweetened desserts, fried food, processed meat, refined grain and high-fat dairy products) ${ }^{21}$ and Western (high intake of meat pies, processed meats, pizza, chips, hamburgers, refined grains, sugar products and beer ${ }^{23}$ dietary patterns. However, other similar studies have found no such association. ${ }^{22,24,25}$ Further, our study noted no association between a Westernised 
dietary pattern (high intake of meat, processed meat, bread, dairy products, coffee and alcohol) and suicide risk. It has been suggested that inflammatory processes may play a role in the pathogenesis of depression, ${ }^{37,38}$ and some Western studies ${ }^{39,40}$ showed that a Western dietary pattern characterised by a high intake of meat, refined grains and fats was associated with increased inflammation. In contrast, no such association between similar dietary patterns and inflammation was observed in previous Japanese studies, ${ }^{41}$ possibly because of the lower consumption of fat and meat among Japanese compared with Western populations. Moreover, other foods and nutrients contributing to this dietary pattern such as tryptophan (included in meat $)^{19}$ and coffee ${ }^{8,9}$ have been shown to be associated with a decreased risk of suicide. The effect of individual foods might therefore be negated by their simultaneous consumption with other items, cancelling out their effect.

We observed no association between a traditional Japanese dietary pattern, characterised by a high intake of fish and seafood, and suicide risk. Several studies have reported a decreased risk of suicide or suicidal ideation associated with a higher intake of fish or higher level of omega-3 fatty acids. ${ }^{6,42-45}$ In contrast, we previously observed no reduction in suicide risk among those with a higher intake of fish in the same study population. ${ }^{7}$ Similarly, a Finnish study found no association between fish or omega-3 fatty acid intake and suicide. ${ }^{5}$ Although we have no plausible explanation for the inconsistent results, the difference in the amount of fish intake among study populations may account for these findings. If there is a threshold above which fish intake offers no additional benefit to mood then the association with fish intake, or a dietary pattern characterised by fish, may not be observed in populations with a high fish intake, such as the Japanese.

\section{Strengths and limitations}

The strengths of the present study include its large sample size, its population-based prospective design, the relatively few participants lost during follow-up, its use of a validated FFQ and its extensive adjustment for potentially important confounding factors including self-reported mental stress, living status and educational level. Death registration in Japan, which we used to identify the causes of death, is believed to be exhaustive. However, several limitations to the present study also warrant mention.

First, dietary intake was assessed only at one time point, and long-term habits may therefore not be accurately reflected. Repeated assessment of diet over a long period of time before suicide would likely provide a better estimate of the exposure status. Second, mental illness including depression status was not assessed in the JPHC study, and therefore the presence of depression at the time of baseline dietary assessment might have influenced both dietary patterns and future suicide risk. However, we analysed data with exclusion of deaths from suicide during the first 3 years of follow-up to minimise such an influence. Moreover, we observed an association between a prudent dietary pattern and suicide in participants with low or medium mental stress but not in those with high mental stress.

Third, because we had no information on other potential confounding factors including substance misuse and family history of suicidal behaviour, we cannot completely rule out bias because of these confounders. Fourth, the principal component analysis requires subjective decisions in determining the number of factors to retain, choosing the method of rotation of the initial factors and labelling the dietary patterns. ${ }^{20}$ However, we maintain that the validity and reproducibility of the three dietary patterns derived from the subsample was acceptable. ${ }^{31}$ Fifth, participants with a higher score for a traditional Japanese dietary pattern might have better psychosocial environments and lifestyles in terms of healthcare preference and availability, caring by and for family members, use of technology, exercise pattern and religion/faith than those with a lower score. However, we did not obtain such information. Sixth, although changes in economic situation during the follow-up period might have influenced the suicide rate in this cohort, they might not have changed the association between dietary patterns and suicide risk. Seventh, there might have been some reporter bias where participants intentionally or unintentionally gave erroneous information as we used self-administered questionnaires.

Finally, because respondents to the second survey appear to be more health conscious compared with non-respondents, the present finding may not represent the whole study population. In addition, because study participants were aged 45-74 years, caution is required to apply the present findings to younger age groups. Moreover, the healthcare system in Japan, with universal health coverage assuring access to a wide array of health services for the whole population, contrasts with, for example, that in the USA (private system). As the differences in healthcare systems among countries might influence suicide risk, the extrapolation of the present findings to populations with other types of healthcare system may be inappropriate.

\section{Implications}

A prudent dietary pattern, characterised by a high intake of vegetables, fruits, soy products, potatoes, seaweed, mushrooms and fish, was associated with a decreased risk of suicide among Japanese adults. The present findings suggest that a prudent dietary pattern is important in preventing suicide. Further research among populations with different social background will be required to confirm our findings.

Akiko Nanri, PhD, Tetsuya Mizoue, MD, PhD, Department of Epidemiology and Prevention, Center for Clinical Sciences, National Center for Global Health and Medicine, Tokyo; Kalpana Poudel-Tandukar, PhD, Waseda Institute for Advanced Study (WIAS), Waseda University, Tokyo; Mitsuhiko Noda, MD, PhD, Department of Diabetes and Metabolic Medicine, National Center for Global Health and Medicine, Tokyo; Masayuki Kato, MD, PhD, Japan Foundation for the Promotion of International Medical Research Cooperation, Tokyo; Kayo Kurotani, PhD Department of Epidemiology and Prevention, Center for Clinical Sciences, National Center for Global Health and Medicine, Tokyo; Atsushi Goto, MD, PhD, Department of Diabetes and Metabolic Medicine, National Center for Global Health and Medicine, Tokyo; Shino Oba, PhD, Department of Health Promotion, National Institute of Public Health, Saitama; Manami Inoue, MD, PhD, Shoichiro Tsugane, MD, PhD, Epidemiology and Prevention Division, Research Center for Cancer Prevention and Screening, National Cancer Center, Tokyo, Japan

Correspondence: Akiko Nanri, Department of Epidemiology and Prevention, Center for Clinical Sciences, National Center for Global Health and Medicine, 1-21-1 Toyama, Shinjuku-ku, Tokyo 162-8655, Japan. Email: nanri@ri.ncgm.go.jp

First received 11 May 2012, final revision 7 Jun 2013, accepted 18 Jul 2013

\section{Funding}

The study was supported by Grants-in-Aid for Cancer Research (19shi-2) and a Health Sciences Research Grant (Research on Comprehensive Research on Cardiovascular Diseases H19-016) from the Ministry of Health, Labour and Welfare of Japan.

\section{References}

1 World Health Organization. Suicide Prevention (SUPRE). WHO, 2011 (http://www.who.int/mental_health/prevention/suicide/suicideprevent/en/ index.html).

2 Hawton K, van Heeringen K. Suicide. Lancet 2009; 373: 1372-81.

3 World Health Organization. Suicide Rate per 100,000 by Country, Year and Sex (Table). WHO, 2011 (http://www.who.int/mental_health/prevention/ suicide_rates/en/). 
4 Ministory of Health, Labour and Welfare. Vital Statistics of Japan, 2008 [in Japanese]. Health and Welfare Statistics Association.

5 Hakkarainen R, Partonen T, Haukka J, Virtamo J, Albanes D, Lonnqvist J. Is low dietary intake of omega-3 fatty acids associated with depression? Am J Psychiatry 2004; 161: 567-9.

6 Hirayama T. Life-style and Mortality: A Large-Scale Census-Based Cohort Study in Japan. Karger, 1990.

7 Poudel-Tandukar K, Nanri A, Iwasaki M, Mizoue T, Matsushita Y, Takahashi Y, et al. Long chain n-3 fatty acids intake, fish consumption and suicide in a cohort of Japanese men and women-the Japan Public Health Center-based (JPHC) prospective study. J Affect Disord 2011; 129: 282-8.

8 Kawachi I, Willett WC, Colditz GA, Stampfer MJ, Speizer FE. A prospective study of coffee drinking and suicide in women. Arch Intern Med 1996; 156: $521-5$.

9 Klatsky AL, Armstrong MA, Friedman GD. Coffee, tea, and mortality. Ann Epidemiol 1993; 3: 375-81.

10 Tanskanen A, Tuomilehto J, Viinamaki $\mathrm{H}$, Vartiainen $\mathrm{E}$, Lehtonen J, Puska P. Heavy coffee drinking and the risk of suicide. Eur J Epidemiol 2000; 16: 789-91.

11 Akechi T, Iwasaki M, Uchitomi $Y$, Tsugane S. Alcohol consumption and suicide among middle-aged men in Japan. Br J Psychiatry 2006; 188: 231-6.

12 Andreasson S, Romelsjo A, Allebeck P. Alcohol, social factors and mortality among young men. Br J Addict 1991; 86: 877-87.

13 Klatsky AL, Armstrong MA. Alcohol use, other traits, and risk of unnatural death: a prospective study. Alcohol Clin Exp Res 1993; 17: 1156-62.

14 Mukamal KJ, Kawachi I, Miller M, Rimm EB. Drinking frequency and quantity and risk of suicide among men. Soc Psychiatry Psychiatr Epidemiol 2007; 42 153-60.

15 Nakaya N, Kikuchi N, Shimazu T, Ohmori K, Kakizaki M, Sone T, et al. Alcoho consumption and suicide mortality among Japanese men: the Ohsaki Study. Alcohol 2007; 41: 503-10.

16 Paffenbarger Jr RS, King SH, Wing AL. Chronic disease in former college students. IX. Characteristics in youth that predispose to suicide and accidental death in later life. Am J Public Health Nations Health 1969; 59: 900-8.

17 Ross RK, Bernstein L, Trent L, Henderson BE, Paganini-Hill A. A prospective study of risk factors for traumatic deaths in a retirement community. Prev Med 1990; 19: 323-34.

18 Turvey $\mathrm{CL}$, Conwell $\mathrm{Y}$, Jones MP, Phillips $\mathrm{C}$, Simonsick $\mathrm{E}$, Pearson JL, et al Risk factors for late-life suicide: a prospective, community-based study. Am J Geriatr Psychiatry 2002; 10: 398-406.

19 Voracek M, Tran US. Dietary tryptophan intake and suicide rate in industrialized nations. J Affect Disord 2007; 98: 259-62.

20 Hu FB. Dietary pattern analysis: a new direction in nutritional epidemiology Curr Opin Lipidol 2002; 13: 3-9.

21 Akbaraly TN, Brunner EJ, Ferrie JE, Marmot MG, Kivimaki M, Singh-Manoux A. Dietary pattern and depressive symptoms in middle age. $\mathrm{Br} J$ Psychiatry 2009; 195: 408-13.

22 Jacka FN, Mykletun A, Berk M, Bjelland I, Tell GS. The association between habitual diet quality and the common mental disorders in community-dwelling adults: the hordaland health study. Psychosom Med 2011; 73: 483-90.

23 Jacka FN, Pasco JA, Mykletun A, Williams LJ, Hodge AM, O'Reilly SL, et al. Association of Western and traditional diets with depression and anxiety in women. Am J Psychiatry 2010; 167: 305-11.

24 Nanri A, Kimura $Y$, Matsushita $Y$, Ohta M, Sato M, Mishima N, et al. Dietary patterns and depressive symptoms among Japanese men and women. Eur J Clin Nutr 2010; 64: 832-9.

25 Samieri C, Jutand MA, Feart C, Capuron L, Letenneur L, Barberger-Gateau P. Dietary patterns derived by hybrid clustering method in older people: association with cognition, mood, and self-rated health. J Am Diet Assoc 2008; 108: 1461-71.

26 Tsugane S, Sobue T. Baseline survey of JPHC study-design and participation rate. Japan Public Health Center-based Prospective Study on Cancer and Cardiovascular Diseases. J Epidemiol 2001; 11: S24-9.
27 Sasaki S, Kobayashi M, Ishihara J, Tsugane S. Self-administered food frequency questionnaire used in the 5-year follow-up survey of the JPHC Study: questionnaire structure, computation algorithms, and area-based mean intake. J Epidemiol 2003; 13: S13-22.

28 Ishihara J, Sobue T, Yamamoto S, Yoshimi I, Sasaki S, Kobayashi M, et al. Validity and reproducibility of a self-administered food frequency questionnaire in the JPHC Study Cohort II: study design, participant profile and results in comparison with Cohort I. J Epidemiol 2003; 13: S134-47.

29 Sasaki S, Ishihara J, Tsugane S. Reproducibility of a self-administered food frequency questionnaire used in the 5-year follow-up survey of the JPHC Study Cohort I to assess food and nutrient intake. J Epidemiol 2003; 13: S115-24.

30 Sasaki S, Kobayashi M, Tsugane S. Validity of a self-administered food frequency questionnaire used in the 5-year follow-up survey of the JPHC Study Cohort I: comparison with dietary records for food groups. J Epidemiol 2003; 13: S57-63.

31 Nanri A, Shimazu $T$, Ishihara J, Takachi $R$, Mizoue $T$, Inoue $M$, et al. Reproducibility and validity of dietary patterns assessed by a food frequency questionnaire used in the 5-year follow-up survey of the Japan Public Health Center-Based Prospective Study. J Epidemiol 2012; 22: 205-15.

32 World Health Organization. The ICD-10 Classification of Mental and Behavioural Disorders: Clinical Descriptions and Diagnostic Guidelines. WHO, 1992

33 Gilbody S, Lightfoot T, Sheldon T. Is low folate a risk factor for depression? A meta-analysis and exploration of heterogeneity. J Epidemiol Community Health 2007; 61: 631-7.

34 Murakami K, Mizoue T, Sasaki S, Ohta M, Sato M, Matsushita $Y$, et al. Dietary intake of folate, other B vitamins, and omega-3 polyunsaturated fatty acids in relation to depressive symptoms in Japanese adults. Nutrition 2008; 24: $140-7$.

35 Bottiglieri T. Homocysteine and folate metabolism in depression. Prog Neuropsychopharmacol Biol Psychiatry 2005; 29: 1103-12.

36 Bilici M, Efe H, Koroglu MA, Uydu HA, Bekaroglu M, Deger O. Antioxidative enzyme activities and lipid peroxidation in major depression: alterations by antidepressant treatments. J Affect Disord 2001; 64: 43-51.

37 Bremmer MA, Beekman AT, Deeg DJ, Penninx BW, Dik MG, Hack CE, et al. Inflammatory markers in late-life depression: results from a population-based study. J Affect Disord 2008; 106: 249-55.

38 Gimeno D, Kivimaki M, Brunner EJ, Elovainio M, De Vogli R, Steptoe A, et al. Associations of C-reactive protein and interleukin- 6 with cognitive symptoms of depression: 12-year follow-up of the Whitehall II study. Psychol Med 2009; 39: 413-23.

39 Fung TT, Rimm EB, Spiegelman D, Rifai N, Tofler GH, Willett WC, et al. Association between dietary patterns and plasma biomarkers of obesity and cardiovascular disease risk. Am J Clin Nutr 2001; 73: 61-7.

40 Lopez-Garcia E, Schulze MB, Fung TT, Meigs JB, Rifai N, Manson JE, et al. Major dietary patterns are related to plasma concentrations of markers of inflammation and endothelial dysfunction. Am J Clin Nutr 2004; 80: 1029-35.

41 Nanri A, Yoshida D, Yamaji T, Mizoue T, Takayanagi R, Kono S. Dietary patterns and C-reactive protein in Japanese men and women. Am J Clin Nutr 2008; 87: 1488-96.

42 Huan M, Hamazaki K, Sun Y, Itomura M, Liu H, Kang W, et al. Suicide attempt and n-3 fatty acid levels in red blood cells: a case control study in China. Biol Psychiatry 2004; 56: 490-6.

43 Lewis MD, Hibbeln JR, Johnson JE, Lin YH, Hyun DY, Loewke JD. Suicide deaths of active-duty US military and omega-3 fatty-acid status: a case-control comparison. J Clin Psychiatry 2011; 72: 1585-90.

44 Sublette ME, Hibbeln JR, Galfalvy $H$, Oquendo MA, Mann JJ. Omega-3 polyunsaturated essential fatty acid status as a predictor of future suicide risk. Am J Psychiatry 2006; 163: 1100-2.

45 Tanskanen A, Hibbeln JR, Hintikka J, Haatainen K, Honkalampi K, Viinamaki H. Fish consumption, depression, and suicidality in a general population. Arch Gen Psychiatry 2001; 58: 512-3. 